Website: http://revistas.lamolina.edu.pe/index.php/acu/index

(C) Universidad Nacional Agraria La Molina, Lima - Perú

\title{
Análisis temporal del canal de riego Peribuela y su influencia en los sistemas agrícolas de la comunidad
}

\section{Temporal analysis of the Peribuela irrigation canal and its influence on community agricultural systems}

\author{
Juan Pablo Aragón ${ }^{1 *}$, Edith Burbano ${ }^{1}$, José Guzmán ${ }^{1}$, Marcelo Albuja ${ }^{1}$
}

*Autor de correspondencia

\begin{abstract}
Resumen
El objetivo del estudio fue analizar los cambios en los sistemas de producción agrícola generados a través del tiempo con la intervención en la infraestructura del canal. El método aplicado fue Expost, se analizó dos etapas de tiempo: antes del mejoramiento del canal de riego y posterior a la modificación. Se identificaron elementos y factores en los sistemas de producción agrícola, mediante la técnica de entrevista y aplicación de mapas temáticos temporales. Se estudiaron los sistemas de producción agrarios y uso de suelo en las dos etapas de tiempo. Los resultados más relevantes fueron: a) El uso de suelo se mantenía en tres cultivos antes del mejoramiento del canal y se diversificó a 16 cultivos posterior a la intervención. b) Los sistemas de producción agrícola se intensificaron en monocultivos de tomate de árbol, maíz y fréjol en el $69 \%$ del área de influencia del canal. c) El $31 \%$ de 13 cultivos entre frutales y hortalizas. d) Se implementaron 1,5 ha de invernaderos y $4284 \mathrm{~m}^{2}$ de reservorios. Como consecuencia de la implementación del canal se amplió el uso de pesticidas, fertilizantes químicos, empleo de maquinaria y costos de producción; de igual forma, los rendimientos se aumentaron con distintos impactos socioeconómicos y ecológicos.
\end{abstract}

Palabras clave: análisis temporal; sistemas de producción; canal de riego; impacto socioeconómico y ecológico; Ecuador.

\begin{abstract}
The objective of the study was to analyze the changes in the agricultural production systems generated over time with the intervention in the canal infrastructure. The method applied was Expost, two stages of time were analyzed: before the improvement of the irrigation channel and after the modification. Elements and factors were identified in the agricultural production systems, through the technique of interview and application of temporary thematic maps. The agrarian production systems and land use were studied in the two stages of time. The most relevant results were: a) Land use was maintained in three crops before the canal was improved and diversified to 16 crops after the intervention. b) Agricultural production systems were intensified in monocultures of tree tomato, corn and beans in $69 \%$ of the area of influence of the canal. c) $31 \%$ of 13 crops between fruit and vegetables. d) 1.5 ha of greenhouses and $4284 \mathrm{~m} 2$ of reservoirs were implemented. As a consequence of the implementation of the canal, the use of pesticides, chemical fertilizers, use of machinery and production costs were expanded; likewise, the yields were increased with different socioeconomic and ecological impacts.
\end{abstract}

Keywords: temporal analysis; production systems; water channel; socio-economic and ecological impact; Ecuador.

\section{Introducción}

Los objetivos de desarrollo sostenible 2015-2030 y los objetivos mundiales, hacen referencia a la producción agrícola dentro de ecosistemas comunitarios. En el Ecuador estos sistemas, están implementados de acuerdo a factores como rendimiento, condiciones climáticas y principalmente por la disponibilidad del recurso agua (Naranjo 2008). En la comunidad de Peribuela, antes de la implementación del canal de riego (1982) los sistemas de producción se establecieron sobre la base de las condiciones climáticas de la zona.

El incremento demográfico y la creciente demanda de alimentos a nivel mundial motivan a utilizar diferentes sistemas de producción agrícola, siendo uno de ellos, la aplicación del agua de riego de forma técnica. La construcción de un canal de regadío afectó los tipos de cultivos, superficie de siembra, manejo de fertilización, fitosanidad y labores culturales.

La agricultura es una de las actividades con mayor incidencia en la conservación del suelo, agua y aire donde se desarrolla la agro biodiversidad (Sarandón y Flores, 2009). En estudios sobre la conservación del suelo, agua y sus efectos, mencionan que los canales de riego en campos de cultivo es una de medida imperativa de la agricultura para el aprovechamiento eficiente de este recurso. Zapatta y Gasselin (2009) mencionan que en promedio el 70\% del 
agua dulce a nivel mundial se emplea en la agricultura, el $30 \%$ se reparte en la industria y el consumo de los hogares. En Ecuador el consumo de agua para la agricultura es el $72 \%$ que está sobre el promedio mundial.

En América Latina y Ecuador el 70 \% de la población se dedica a actividades agropecuarias (Clements et al., 2013). El manejo del agua en el campo debe ser eficiente y responsable, el uso inadecuado en la producción agrícola conlleva a impactos negativos como degradación de suelos, contaminación de agua y del aire que afecta los cultivos y la alimentación del ser humano.

A nivel mundial los países desarrollados invierten en tecnologías para el mejor uso del agua y sistemas de producción agrícola más eficientes, esto genera impactos sobre la agro economía de cada país.

En América Latina es similar situación, los países más avanzados como Brasil, Chile, Argentina, México son quienes emplean el agua con mayor eficiencia. Además, lideran las tecnologías en sistemas de producción agrícolas que generan mayores ingresos a los agricultores (FAO, 2015b).

En el Ecuador, el balance hídrico global del país es positivo, incluso por vertiente, pero existen cuencas deficitarias en varias zonas y en algunas épocas del año como en las provincias de Manabí, Loja y El Oro, las cuales necesitan implementar canales de riego (Secretaría Nacional del Agua SENAGUA, 2013). En el año 2013 existía un millón y medio de hectáreas con regadío, de las cuales el $81 \%$ estaban dedicadas a la producción agropecuaria. (Ministerio de Agricultura, Ganadería, Acuacultura y Pesca, 2013).

En el Ecuador la economía de los sistemas de producción agrícola contribuye a objetivos de gobierno como la soberanía alimentaria, y aporta a objetivos del Desarrollo Sostenible, como: erradicar la pobreza extrema y la conservación del medio ambiente. (Organización de la Naciones Unidas para la Alimentación y Agricultura, 2015a).

El MAGAP (2013) hace referencia a que los sistemas de producción agrícolas deben ser más eficientes en el manejo del recurso agua. La conservación de los recursos naturales, suelo y agua son indispensables para la producción agraria de la comunidad y deben garantizar la seguridad alimentaria del sector.

No existe información sobre cambios en la agricultura generados por la implementación de un canal de riego, por tanto, es importante la identificación y análisis de factores que se han transformado a través del tiempo.

\section{Materiales y métodos}

La presente investigación se realizó en la microcuenca del canal de riego Peribuela perteneciente a la cuenca del río Ambi, parroquia Imantag, cantón Cotacachi, provincia de Imbabura (Ilustre Municipio de Santa Ana de Cotacachi, 2011).

\section{CANAL DE RIEGO PERIBUELA - UBICACIÓN BOCATOMA DEL CANAL DE RIEGO}
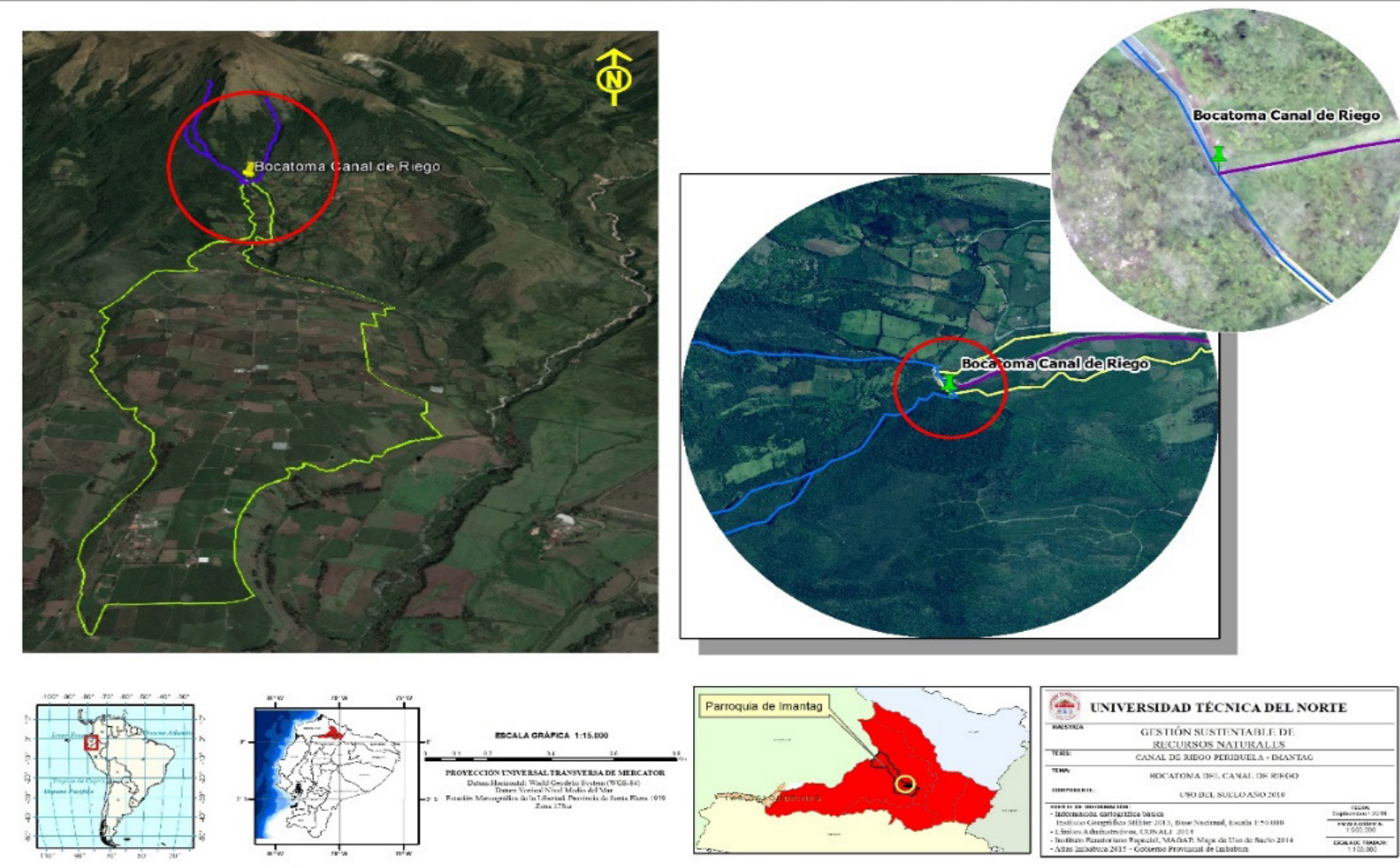

Figura 1. Área de influencia de la microcuenca del canal de riego Peribuela 
Se aplicó investigación descriptiva, para analizar las características agronómicas en la microcuenca del canal antes y después de su intervención (Sampieri et al., 2010). Se dividió en tres fases:

FASE I: Se realizó una caracterización agronómica en el área de influencia de la microcuenca del canal de riego Peribuela mediante Sistemas de Información Geográfica en el ciclo diciembre 2016 a enero 2017. La misma información se obtuvo del año 1982 antes de la intervención sobre la infraestructura del canal. FASE II: Se identificó factores que cambiaron desde el año 1982 al 2017, período en el cual el canal de riego tuvo modificaciones. FASE III: Se analizó los factores identificados antes y después de la intervención del canal, para evaluar estos dos momentos de tiempo. Para determinar el uso de suelo de los años 1982 y 2016-2017, se utilizó mapas temáticos con software argis 10.3 Se aplicó encuestas dirigida a los regantes con un cuestionario sobre situación de la finca, manejo y uso de suelo, y preguntas de percepción mediante escala Likert.

\section{Resultados y discusión}

En el año 2017 el canal de riego tenía un área de influencia de 342 ha y una cobertura de entre $2900 \mathrm{msnm}$ donde se origina el tramo Peribuela, y finaliza a los $2470 \mathrm{msnm}$. Atraviesa un terreno escarpado con una longitud de 5,7 $\mathrm{Km}$ en una pendiente del $26 \%$ y orientación de oeste a este. Da servicio a 119 usuarios, dedicados a la agricultura.

En el período 2016-2017, la microcuenca del canal de riego Peribuela tiene la siguiente distribución de suelo: 238 ha equivalente al $69 \%$ de la superficie total regada, con sistema de monocultivo en tomate de árbol Solanum betaceum), maíz (Zea mays) y fréjol (Phaseolus vulgaris). El $31 \%$ de la superficie restante, está distribuida entre trece cultivos (Tabla 1). En el año 1982 son dos los cultivos principales: trigo y cebada, ocupando el 35 $\%$ de la superficie, y el otro $65 \%$ cubierta por arbustos. Determinándose que los sistemas de producción agrícola se incrementaron en 234 ha para el año 2017.

Con el mejoramiento del canal a través del proyecto Rehabilitación de la infraestructura de riego y fortalecimiento de los Juntas de Agua El Morlán, Colimbuela, Quitubí y Peribuela del cantón Cotacachi, ejecutado en el año 2007 por parte de la Secretaría Nacional del Agua (SENAGUA), los cultivos descritos en la tabla 1 , se establecieron por el incremento de disponibilidad de agua, cumpliendo con el objetivo de mejorar la productividad de los usuarios, debido a la eficiencia en la conducción del recurso (SENAGUA, 2014). A enero del año 2017 hay cuatro reservorios de la comunidad que ocupa $4284 \mathrm{~m}^{2}$ de superficie, de los cuales se desprenden ramales para mini estanques en algunas fincas a diferencia del año 1982 que no había ningún reservorio, porque la demanda de agua de los cultivos no requería. El $84 \%$ de los cultivos cuentan con un sistema de riego por gravedad y el $16 \%$ por goteo y microaspersión; este último, básicamente para cultivo de tomate hortícola (Solanum licopersicum) bajo invernadero y aguacate (Persea americana). De las 342 ha con regadío, únicamente 1,5 ha están bajo invernadero. La infraestructura vial adicional hasta el año 2017 son 13,67 Km de camino de tercer orden que conecta las fincas con una dirección referencial del canal de riego (Figura 2), mientras que en 1982 no existían vías de tercer orden porque los sistemas de producción agrícolas no se encontraban desarrollados.

Tabla 1. Distribución de cultivos en el área influenciada por el canal de riego Peribuela, año 2016.

\begin{tabular}{|c|c|c|}
\hline Tipo de cultivo & $\begin{array}{l}\text { Superficie } \\
\text { Ha }\end{array}$ & Detalle \\
\hline Aguacate & 7,8 & $\begin{array}{l}\text { Superficie directamente } \\
\text { beneficiada por el canal de riego. }\end{array}$ \\
\hline $\begin{array}{l}\text { Babaco (Carica } \\
\text { pentágono })\end{array}$ & 0,6 & $\begin{array}{l}\text { Cultivo no tradicional que está } \\
\text { ingresando en la comunidad. }\end{array}$ \\
\hline Barbecho & 23,38 & Terrenos para futuras siembras. \\
\hline Fréjol & 45 & $\begin{array}{l}\text { Cultivo tradicional de la } \\
\text { comunidad. }\end{array}$ \\
\hline Hortalizas & 0,68 & $\begin{array}{l}\text { Cultivos no tradicionales para } \\
\text { consumo familiar. }\end{array}$ \\
\hline Maíz & 147,32 & Principal cultivo del sector. \\
\hline Tomate de árbol & 42,28 & $\begin{array}{l}\text { Principal cultivo y el más } \\
\text { rentable. }\end{array}$ \\
\hline Terreno preparado & 7,44 & Por sembrar. \\
\hline Bosque & 44,55 & $\begin{array}{l}\text { Que es influenciado por el canal } \\
\text { de riego. }\end{array}$ \\
\hline $\begin{array}{l}\text { Reservorios, } \\
\text { invernaderos, } \\
\text { quebradas, } \\
\text { caminos, } \\
\text { infraestructuras, } \\
\text { entre otros } \\
\end{array}$ & 23 & $\begin{array}{l}\text { Existen construcciones, } \\
\text { accidentes geográficos que están } \\
\text { dentro del área de influencia del } \\
\text { canal de riego. }\end{array}$ \\
\hline
\end{tabular}

La implementación de nuevos cultivos por mayor disponibilidad de agua conduce a la expansión de la frontera agrícola y disminución de áreas forestales. En la comunidad de Peribuela el principal efecto del canal de riego se evidencia en: incremento de cultivos de mayor productividad, alto riesgo de salinización en suelos por abuso de fertilizantes sintéticos, incremento de uso de pesticidas que inciden en la biodiversidad microbiana del suelo, y maquinaria agrícola que afecta la estructura y compactación del suelo (Tabla 2). Estos cambios, también los describe Padilla et al. (2015) en su trabajo investigativo sobre Adaptación y mitigación del impacto del cambio climático en México. Mientras que sin canal de riego los sistemas de producción agrícolas no causaban impactos importantes a los recursos naturales ni culturales (alteración de los ciclos de siembra habituales) de la comunidad.

Los cultivos predominantes en el año 2016 y 2017 son: tomate de árbol, maíz y fréjol; y, los principales del año 1982 son trigo y cebada. El costo de producción está en proporción directa a la aplicación de fertilizantes y pesticidas sintéticos, la productividad también está en la misma relación. Al analizar el aspecto económico, son más rentables los cultivos sustitutos (tomate de árbol, maíz y fréjol) que los tradicionales (trigo y cebada). 


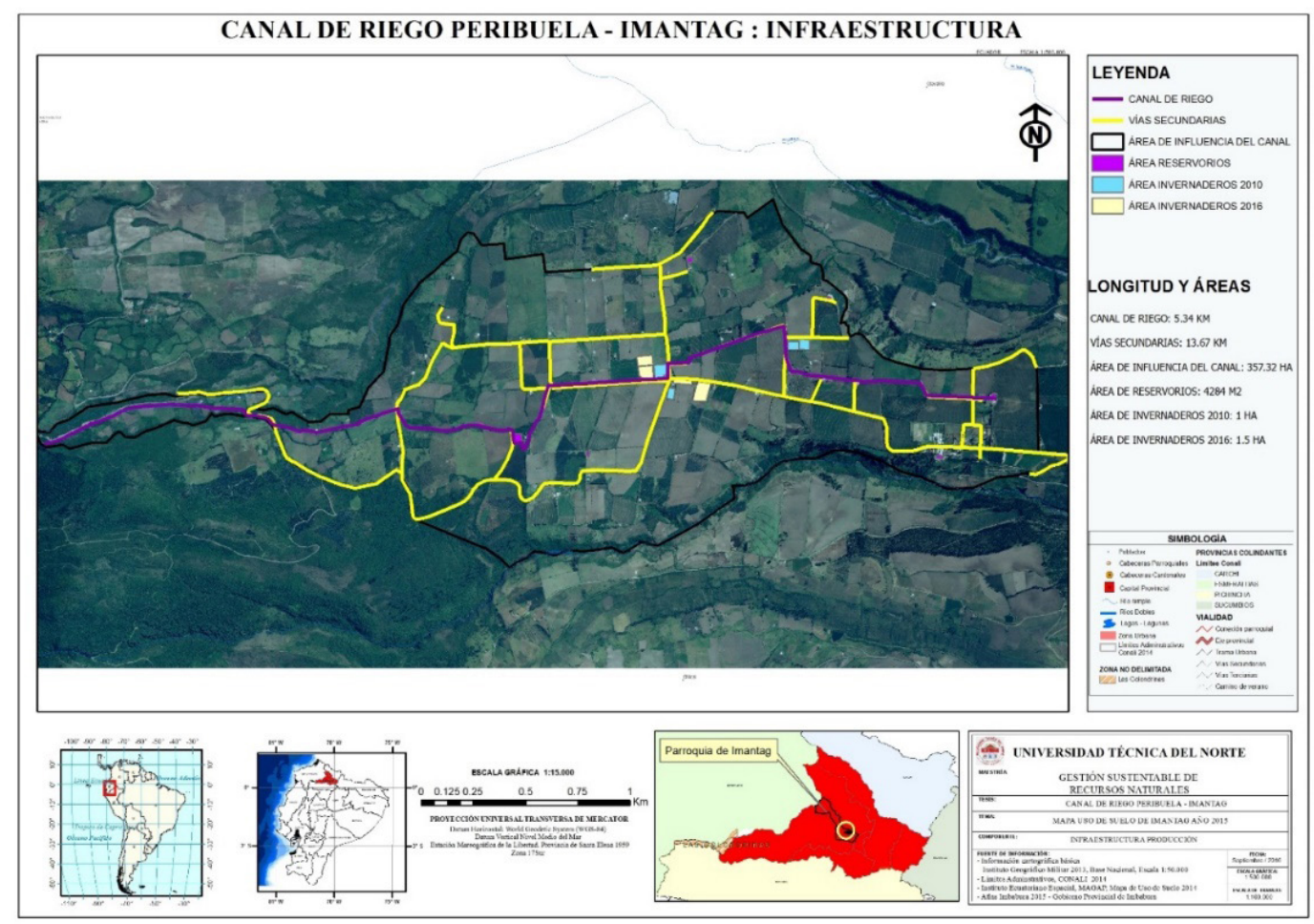

Figura 2. Infraestructura existente en el área de influencia del canal de riego de Peribuela

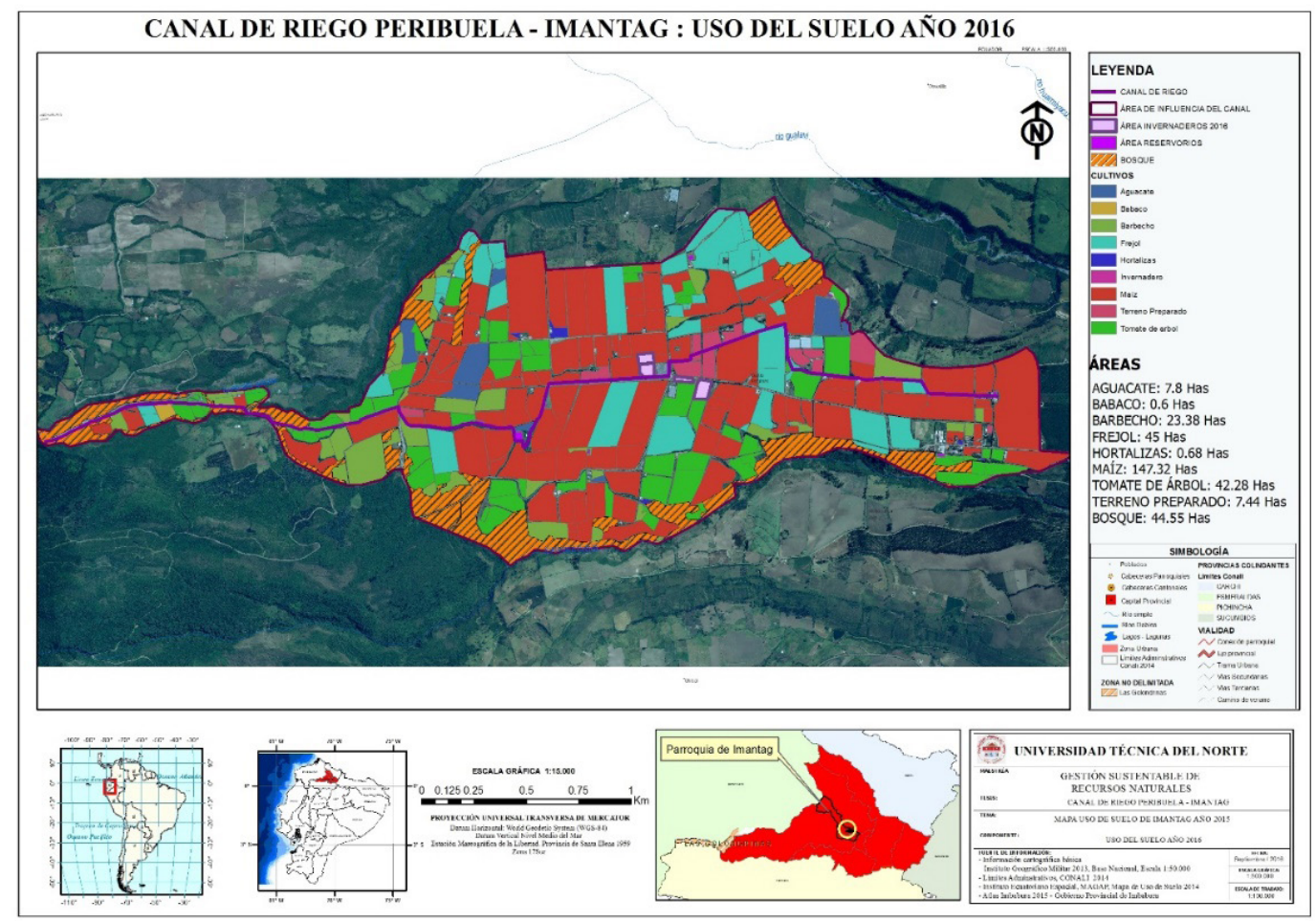

Figura 3. Uso del suelo en la microcuenca del canal de riego Peribuela 2016-2017

El uso de suelo es otro factor relevante en los cambios del ecosistema a partir de la intervención en el canal. En la Figura 3 se identifica que en el ciclo 2016-2017 hay alrededor de 16 tipos de cultivos diferentes.

En el año 1982, existió predominio de dos cultivos (trigo y cebada) en una área de 212 ha y las 236 ha restantes estaba cubierta de arbustos, como se evidencia en la Figura 4.

A mayor disponibilidad de agua para las labores culturales, el rendimiento en los cultivos implementados 
fue de mayor productividad y más rentables. De igual forma en la Tabla 2, muestra que los costos y la productividad varían en función de los cambios en el canal de riego.

Esta variación en el uso de suelo promueve el incremento de la diversificación de cultivos, aspecto positivo porque cuentan con más especies agrícolas y albergan un sinnúmero de especies menores, insectos y microorganismos. Sin embargo, un aspecto negativo, es el incremento de

la expansión agrícola generado por la deforestación y degradación de ecosistemas (Larrea et al., 2015).

Para mayor eficiencia del uso del agua, la comunidad estableció frecuencia, tiempo y caudal de riego, determinante en la evolución de los sistemas de producción agrícola de la comunidad Peribuela (Tabla 3).

Tabla 3 Gestión de riego en la comunidad Peribuela

\begin{tabular}{cccc}
\hline $\begin{array}{c}\text { Caudal } \\
\text { Canal }\end{array}$ & $\begin{array}{c}\text { Caudal por } \\
\text { usuario }\end{array}$ & $\begin{array}{c}\text { Frecuencia de } \\
\text { riego }\end{array}$ & $\begin{array}{c}\text { Tiempo de } \\
\text { riego/Ha }\end{array}$ \\
\hline $51 \mathrm{l} / \mathrm{s}$ & $15 \mathrm{l} / \mathrm{s}$ & $15 \mathrm{a} 18$ días & $3 \mathrm{a} 4$ horas \\
\hline
\end{tabular}

Tabla 2 Costos de producción, rendimientos y aplicación de insumos sintéticos de cultivos sustitutos y sustituidos en la comunidad de Peribuela

\begin{tabular}{|c|c|c|c|c|c|c|}
\hline Cultivo & $\begin{array}{l}\text { Costo de } \\
\text { producción } \\
\text { usd/Ha }\end{array}$ & $\begin{array}{c}\text { Rendimiento } \\
\mathrm{Tm} / \mathrm{Ha}\end{array}$ & $\begin{array}{c}\text { Cantidad de } \\
\text { fertilización } \\
\text { química en } \\
\mathrm{Kg} / \mathrm{Ha}\end{array}$ & $\begin{array}{c}\text { Número de } \\
\text { tanques con } \\
\text { pesticida ( } 200 \\
\text { lt) }\end{array}$ & $\begin{array}{l}\text { Horas } \\
\text { Tractor } \\
\text { (horas/ } \\
\text { Ha) }\end{array}$ & $\begin{array}{c}\text { Relación } \\
\text { B/C }\end{array}$ \\
\hline $\begin{array}{l}\text { Tomate } \\
\text { de árbol }\end{array}$ & 12,000 & 35 & 1600 & 70 & 20 & 3,5 \\
\hline Maíz & 1,300 & 6 & 700 & $10-12$ & 10 & 3,2 \\
\hline Fréjol & 1,250 & 3 & $600-700$ & $10-12$ & 10 & 4,08 \\
\hline Trigo & 900 & 3 & 450 & 5 & 5 & 1,66 \\
\hline Cebada & 900 & 3 & 450 & 5 & 5 & 1,33 \\
\hline
\end{tabular}

\section{Conclusiones}

Los cultivos tradicionales (trigo y cebada) fueron reemplazados, principalmente, por tomate de árbol, maíz, fréjol, aguacate, babaco, tomate hortícola. Las 234 ha de arbustos fueron sustituidas por los cultivos señalados anteriormente, entre otros. La ejecución del canal tuvo un impacto al modificarse el uso de suelo de 3 a 16 cultivos con todo el sistema de producción que genera cada uno de ellos, como la construcción de 1,5 ha de invernadero y $4284 \mathrm{~m}^{2}$ de reservorios, que antes no existía. La intervención en la infraestructura para el abastecimiento de un recurso natural a través del tiempo, afecta los sistemas de producción agrícola de forma positiva y negativa.

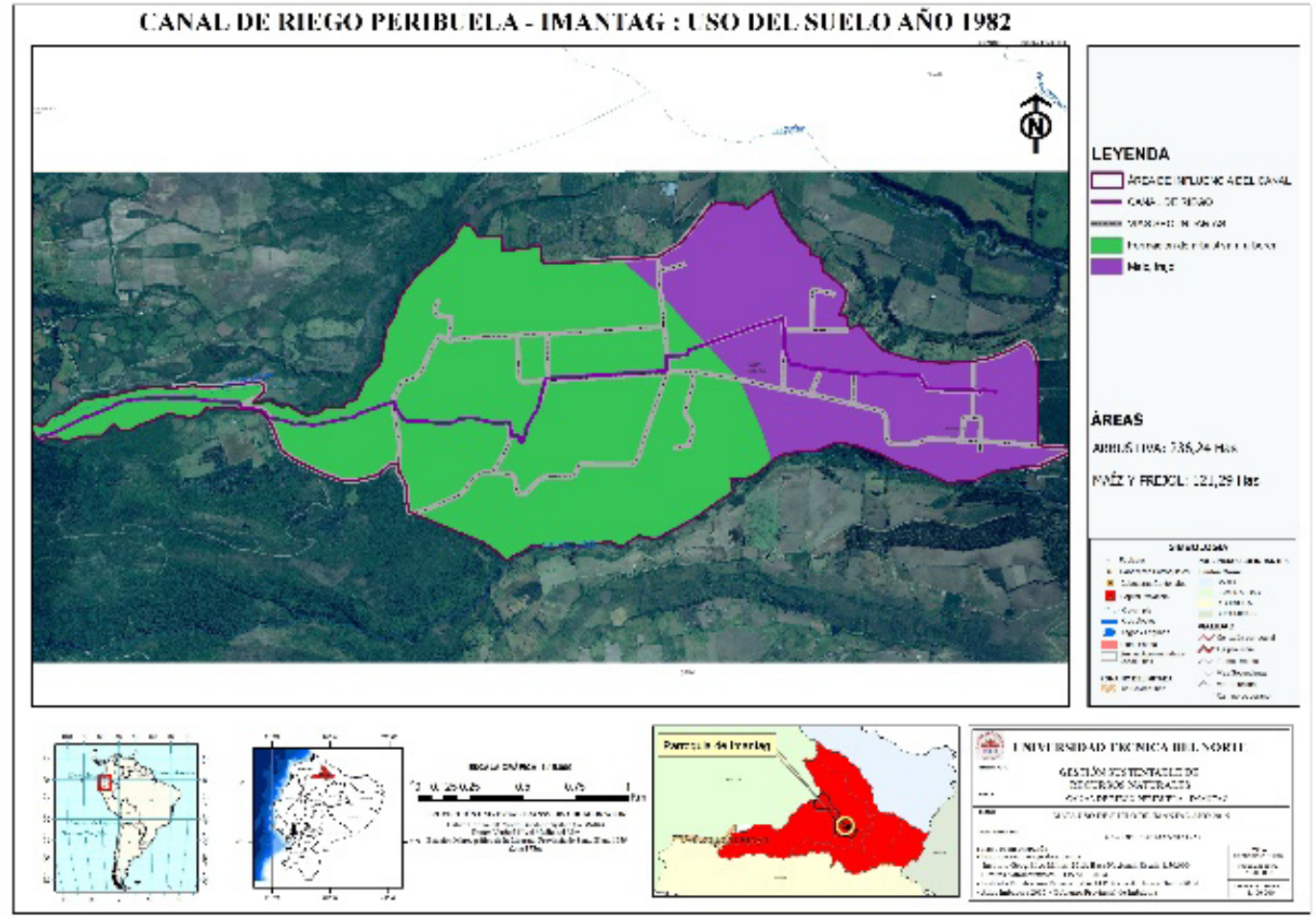

Figura 4. Uso de suelo en la microcuenca del canal de riego Peribuela 1982 


\section{Agradecimientos}

A la Facultad de Ingeniería en Ciencias Agropecuarias y Ambientales de la Universidad Técnica del Norte y los usuarios del canal de riego Peribuela.

\section{Literatura citada}

Clements, R., Haggar, J., Quezada A. y Torres, J. 2013. Tecnologías de Adaptación al Cambio ClimáticoSector Agropecuario-. GEF-PNUMA.

Ilustre Municipio de Santa Ana de Cotacachi. 2011. Plan de Desarrollo y Ordenamiento Territorial 2012-2032.

Larrea, C., Chalem, X., Merino-Viteri, A., Cuesta F., RíosTouma B., Tapia, C., Encalada, A., Utreras V., Peralvo, M., Greene, N., Zapata, G., Iturralde, P., Suárez, E. y Bravo, A. 2015. Propuesta de Indicadores Nacionales de Biodiversidad: una contribución para el sistema nacional de monitoreo del patrimonio natural y para la evaluación del impacto de la implementación de la Estrategia Nacional de Biodiversidad y su Plan de Acción 2015-2020. MAE, CONDESAN, GIZ, PNUD FMAM, UASB. Quito, Ecuador.

Ministerio de Agricultura, Ganadería, Acuacultura y Pesca. 2013. Disponible en: http://www.agricultura. gob.ec/tecnicos-del-magap-se-capacitaron-en-cambioclimatico/

Naranjo, M. 2008. Ecuador: análisis de la contribución de los programas sociales al logro de los Objetivos del Milenio.

FAO [Organización de las Naciones Unidas para la Alimentación y la Agricultura]. 2015a. Sistemas de producción Agropecuaria-Pobreza. Disponible en: http://www.fao.org/farmingsystems/description_es.htm

FAO [Organización de las Naciones Unidas para la Alimentación y la Agricultura]. 2015b. Cambio Climático. Escuelas de campo y de vida para jóvenes agricultores. Roma.

Padilla, L. M., Puebla, J. A. M., y Ceballos, S. E. 2015. Orden jurídico e institucional para la adaptación y mitigación del impacto del cambio climático sobre los humedales costeros del sur de Tamaulipas, México Política y Jurídica, 2(3).

Sampieri, R., Fernández, C. y Baptista, M. 2010. Metodología de la Investigación. Quinta Edición. Mcgraw-Hill / Interamericana Editores, s.a. de c.v. México.

Sarandón, S. J., y Flores, C. C. 2009. Evaluación de la sustentabilidad en agroecosistemas: una propuesta metodológica. Agroecología, 4, 19-28.

Secretaría Nacional del Agua SENAGUA. 2013. Gestión de recursos hídricos en el Ecuador Demarcación hidrográfica de Puyango Catamayo.

SENAGUA [Secretaría Nacional del Agua]. 2014. Calidad del agua en el Ecuador 2011 . Estado situacional del Ecuador en cuanto al manejo de los recursos hídricos 2011. Oferta y demanda hídrica en Ecuador. Disponible en: http://www.senagua.gov.ec

Zapatta, A. y Gasselin, P. 2009. El Riego en el Ecuador: Problemática, Debate y Políticas. Quito-Ecuador. 